\title{
Cateterismo Cardíaco Intervencionista na Cardiologia Pediátrica. O Posicionamento Médico Quanto às Aplicações Atuais e Perspectivas
}

\author{
Edmar Atik
}

São Paulo, SP

Paralelamente ao progresso inestimável da cardiologia pediátrica, assiste-se, hoje, nessa especialidade a uma verdadeira revolução no âmbito diagnóstico e na conduta em geral. Assim, a ecocardiografia firma-se como o exame diagnóstico de eleição na confirmação da suspeita clínica, substituindo o estudo hemodinâmico e angiográfico e, estes, por seu lado, cada vez mais e de maneira apropriada, substituem a cirurgia cardíaca, no alívio de muitos distúrbios hemodinâmicos ${ }^{1-13}$.

Dessa maneira, percebe-se que as fronteiras da especialidade sofreram mudanças e os marcos antigos, os clínicos, hemodinâmicos, cirúrgicos e anatomopatológicos, agora, são certamente acrescidos de outros marcos, dada a intromissão dos métodos entre si, tanto no diagnóstico quanto na conduta.

Ilustrando e acompanhando a evolução dos diferentes campos da especialidade, nos marcos antigos clínicos, semiológicos, da sistematização do conhecimento e dos aspectos clínicos, acresce-se hoje a ajuda ecocardiográfica. Nos marcos hemodinâmicos, acrescenta-se o cateterismo intervencionista e nos cirúrgicos, além da melhoria da perfusão e proteção miocárdicas, as técnicas mais apropriadas e definitivas.

Especificamente, no campo do cateterismo intervencionista, este progresso ocorreu em pouco tempo, se considerarmos que o início dessa mudança substancial tenha ocorrido com a atriosseptostomia de Rashkind em 1966, na transposição das grandes artérias ${ }^{1}$. A feitura da comunicação interatrial, pela mesma técnica, estende-se hoje a outras anomalias como à atresia pulmonar com septo ventricular íntegro, à atresia tricúspide, à atresia da valva atrioventricu-

Instituto do Coração do Hospital das Clínicas - FMUSP

Correspondência: Edmar Atik - InCor - Av. Dr. Enéas C. Aguiar, 44 - 05403-000

- São Paulo, SP - E-mail: conatik@incor.usp.br

Recebido para publicação em 18/10/01

Aceito em 11/12/01 lar esquerda, à estenose mitral, dentre outras. Ainda mais, o método ampliou seus horizontes e com efetividade, tanto no alívio de defeitos obstrutivos, tipo estenoses aórtica e pulmonar, e na coartação da aorta, quanto no fechamento de defeitos septais, tipo comunicação interatrial e ventricular, canal arterial e em tantas outras situações, até então inimagináveis.

A década de 1980 foi batizada como a era dos balões para a feitura de atriosseptostomia e de valvuloplastias, e a década de 1990, por sua vez, a era das endopróteses para fechamento de defeitos com umbrelas e coils e também para dilatar estruturas através do stent.

Nesta ampla aplicação, obteve o cateterismo intervencionista suficiente credibilidade, principalmente pela efetividade do procedimento e a um custo aceitável a ponto de em muitas anomalias poder ser aplicado à população mais carente em verdadeiro favorecimento social, o que consolida o método como opção terapêutica eficaz.

Na prática, quando da preferência pelo cateterismo intervencionista, observa-se em pacientes submetidos ao método a nítida eliminação de distúrbios psicológicos que obrigatoriamente acometem aqueles operados, o que certamente se estende aos familiares, tornando assim menos traumática a conduta. Por esse prisma, sente-se que a correção cirúrgica pode ser seguramente substituída, além do que o grau da credibilidade excedeu as expectativas, mesmo em intervenções sobre obstruções venosas, sistêmicas e pulmonares, em estenoses subaórticas e de colaterais sistêmico-pulmonares, citando alguns exemplos.

Tornou-se o método até superior à operação, dada a efetividade e com eliminação de riscos, em estenoses das artérias pulmonares, em especial das estenoses periféricas intraparenquimatosas, mas também nas obstruções em artérias principais, além da execução das embolizações arteriais referentes à circulação colateral sistêmico-pulmonar e à seqüestração pulmonar, em vasos arteriais que se originam da aorta descendente ou mesmo da aorta abdominal abaixo do diafragma. 
Observa-se também na prática maior liberação de indicação, quando se considera o cateterismo intervencionista, em fechamento de defeitos pequenos cujo temor cirúrgico reside na correção de pacientes assintomáticos e em ampla atividade psicossocial. Assim, a ausência de sintomas torna difícil a visualização da necessidade da operação cardíaca, por exemplo, em comunicação interatrial e em canal arterial silencioso, ambos de discretas dimensões.

Esta última aplicação tornou a indicação pelo cateterismo mais precoce ainda, eliminando os fatores adversos adquiridos como a sobrecarga de volume e/ou de pressão, que certamente obscurecem os resultados, em face de freqüentes postergações das operações corretivas ${ }^{3,9,12}$.

Outro aspecto positivo que deve ser ressaltado é que, indiretamente, o cateterismo intervencionista contribuiu para a melhoria das técnicas operatórias, através especificamente do advento da minitoracotomia, em face da competição obrigatória dos métodos. A diminuição da incisão cirúrgica, hoje tão aceita e aplicada, apresenta dados pós-operatórios semelhantes aos do método intervencionista quanto ao tempo de recuperação, à permanência hospitalar e à ausência de complicações. Tal panorama tem ocorrido rotineiramente após a correção das comunicações interatrial e ventricular, do canal arterial e do próprio defeito do septo atrioventricular.

As vantagens atuais do cateterismo intervencionista relacionam-se ao aperfeiçoamento maior das próteses, à efetividade comprovada do procedimento e à ausência de complicações graves a ponto de ter se tornado opção válida semelhante à intervenção operatória. Outras vantagens devem ser invocadas, como a eliminação da anestesia geral, em muitas ocasiões, a eliminação de efeitos deletérios ao organismo como o provocado pela circulação extracorpórea, pela proteção miocárdica deficiente, pela anóxia cardíaca, além da incisão atrial e pericárdica responsáveis por surgimento de arritmias, de pericardites, de síndromes pós-pericardiotomias, que aumentam certamente a morbidade pósoperatória.

Mais vantagens poderiam ser lembradas na execução de procedimentos pelo cateterismo cardíaco, como o tempo de internação mais curto e o retorno mais precoce do paciente às atividades de rotina.

Por isso, pode-se assim sugerir até que, na correção dos defeitos cardíacos, haja uma divisão de responsabilidades entre a cirurgia e o cateterismo cardíaco intervencionista. Espaço amplo à indicação cirúrgica àquelas crianças que necessitem realmente da correção operatória como em cardiopatias complexas, na transposição das grandes artérias, na drenagem anômala total das veias pulmonares, dentre outras. Indicação ao cateterismo intervencionista em situações já mencionadas nas quais o resultado se mostre similar ao da cirurgia.

Esta orientação e posicionamento se devem ao fato de se verificar resultados semelhantes entre os métodos para fechamento da comunicação interatrial e do canal arterial, por exemplo, com índices idênticos de shunt residual, transcorridos alguns meses das correções. Sucedeu tal fato em vista da evolução das próteses, desde a de Clamshell até a mais atual, de Amplatzer para o fechamento da comunicação interatrial e até as simples molas de Gianturco no fechamento do canal arterial ${ }^{2,4,6,10,12}$. Em ambas as técnicas, as lesões residuais se eqüivalem às verificadas por procedimentos cirúrgicos convencionais e pelo cateterismo intervencionista, em cerca de 1 a $2 \%$ dos casos.

Por isso, o método intervencionista é considerado uma evolução médica e pode substituir a cirurgia em muitas alterações hemodinâmicas. O método se firmou em vista dos resultados adequados e do confronto com os resultados apresentados pela cirurgia.

Pelo progresso obtido, verdadeiramente, assiste-se hoje nas intervenções na cardiologia pediátrica à integração dos métodos, do cateterismo intervencionista e da cirurgia, em um complemento de procedimentos e a uma interação em prol da diminuição dos riscos. Obtêm-se tais efeitos através da melhora das condições pré-operatórias e através da facilitação da correção cirúrgica, utilizando-se em um primeiro tempo a atuação do cateterismo intervencionista ${ }^{5,13}$.

Alguns exemplos dessa atuação podem ser citados como na drenagem anômala total das veias pulmonares, na forma obstrutiva, no tronco arterial comum com estenose da valva truncal, na atresia pulmonar com ventrículo direito hipoplásico, na anomalia de Ebstein com estenose pulmonar. Na primeira dessas anomalias, através da dilatação pelo cateter balão da veia pulmonar obstruída, a hipertensão venocapilar pulmonar diminui e há sensível melhora clínica para correção operatória posterior, em melhores condições. Nas outras anomalias, o alívio da estenose pulmonar valvar acarreta maior facilidade de correção posterior do tronco arterial, da execução de operação paliativa tipo BlalockTaussig sem o uso da circulação extracorpórea na atresia pulmonar e postergação da correção da anomalia de Ebstein a uma faixa etária maior, sob menor risco operatório. Ainda mais, o alívio de gradientes subaórticos na estenose subaórtica com conseqüente diminuição da pressão intraventricular esquerda, da pressão diastólica final ventricular com nítida melhora das condições pré-operatórias, claramente interferindo nos resultados cirúrgicos, pode ser outra aplicação desta interação dos métodos ${ }^{7}$.

Esta interação é essencial para o manejo mais adequado de pacientes com lesões simples e mesmo complexas, planejando-se nestas a cirurgia em melhores condições hemodinâmicas após alívio de obstruções pelo cateter-balão, sendo o cateterismo intervencionista indicado mesmo após modificações de técnicas cirúrgicas, como por exemplo, para a execução de embolização pós-operatória de vasos colaterais sistêmico-pulmonares encontrados na atresia pulmonar com comunicação interventricular.

Neste panorama, tornou-se responsabilidade do clínico não obstaculizar e sim orientar à feitura do tratamento intervencionista pelo cateterismo cardíaco, quando indicado ${ }^{13}$.

Para tal, deve-se conhecer hoje que as indicações para angioplastia com balão, stents e molas, aceitas pela American Heart Association ${ }^{1}$ apresentam-se com resultados apropriados, satisfatórios e inadequados e dessa análise pode-se melhor orientar o paciente. 
Sabe-se, assim, que a angioplastia por cateter-balão é apropriada em estenose valvar pulmonar, na estenose aórtica do recém nascido, na recoartação da aorta, na estenose de veias cavas, na estenose de artérias pulmonares; é satisfatória em estenose de anastomose sistêmicopulmonar, na coartação da aorta nativa acima de sete meses de idade e no canal arterial restritivo de cardiopatias canal arterial dependentes; e é inadequada em estenose de veias pulmonares.

A colocação de stent é apropriada em estenose de artérias pulmonares e em estenose de veias cavas; é satisfatória em estenose de condutos entre o ventrículo direito e as artérias pulmonares, na estenose de colaterais sistêmicopulmonares, na coartação da aorta, e em canal arterial restritivo em cardiopatias canal arterial dependentes; é inadequada em estenose de veias pulmonares.

A inserção de "molas" tornou-se apropriada em colaterais sistêmico-pulmonares na atresia pulmonar e comunicação interventricular, no canal arterial com diâmetro inferior a $4 \mathrm{~mm}$, em anastomoses sistêmico-pulmonares, fístulas arteriovenosas pulmonares e em conexões venovenosas após a operação de Glenn bidirecional; é satisfatória em canal arterial de diâmetros entre $4 \mathrm{e} 7 \mathrm{~mm}$ e em fístulas coronário-cavitárias; é inadequada em canal arterial de grandes dimensões superior a $7 \mathrm{~mm}$ e em grandes colaterais sistêmico-pulmonares.

Em todo esse posicionamento, reconhece-se hoje o cateterismo intervencionista como procedimento de primeira escolha nas estenoses valvares, na recoartação da aorta, para a oclusão de vasos colaterais sistêmico-pulmonares e em estenoses de artérias pulmonares, principais e periféricas. Sendo um método efetivo e seguro, passa a ser opcional à correção operatória no fechamento de defeitos, como nas comunicações interatrial e ventriculare, também, no canal arterial ${ }^{1,11}$.

A evolução certamente surge do confronto de idéias, novas e antigas, além dos resultados. O progresso indiscutível que hoje vivemos através dos avanços do cateterismo cardíaco, talvez, se estendam a caminhos ainda mais fantásticos, servindo este método, quem sabe, até para fechar defeitos por meios gênicos. Este passo promissor tornará real um sonho desejado e almejado quando a terapêutica gênica for efetivamente aplicável. Vale lembrar, como um dos grandes avanços e já real do cateterismo intervencionista, a colocação de válvulas biológicas, armadas em stent, inseridas na altura do anel pulmonar a fim de diminuir a insuficiência valvar pulmonar, tornando-se uma conduta muito promissora em pacientes operados para a correção da tétrade de Fallot ${ }^{8}$.

Nos últimos 50 anos houve uma avalanche de novas técnicas e entendimentos mais profundos no que respeita à etiopatogenia, à fisiopatologia, à clínica, à terapêutica e às possibilidades cirúrgicas. Hoje, os especialistas combatem e previnem a doença cardiovascular mais adequadamente. Sabe-se quando e porque a placa se forma na artéria, quando a fibra cardíaca enfraquece, quando a cavidade atrial fibrila e quando o músculo cardíaco morre.

Combatendo os meios químicos, mecânicos e elétricos, causas comuns de morte, interfere-se e interrompe-se a cadeia de eventos que, certamente, se direcionam à cura das doenças e neste caminho, certamente, o cateterismo cardíaco já se inseriu. Neste contexto, saliento a participação do Dr. Valmir Fernandes Fontes, cujo trabalho perseverante e pioneiro resultou o progresso inestimável do cateterismo intervencionista em nosso meio.

\section{Referências}

1. Allen HD, Driscoll DJ, Fricker FJ, et al. Guidelines for pediatric therapeutic cardiac catheterization. A statement for health professionals from the Committee on Congenital Cardiac Defects of the Council on Cardiovascular Disease in the Young, American Heart Association. Circulation 1991; 84: 2248-58.

2. Chamié F, Pereira SD, Sbaffi F, Serra Jr A, de Athayde JG. Fechamento do canal arterial com molas de Gianturco. Arq Bras Cardiol 1996; 67: 23-7.

3. Fontes VF, Esteves CA, Braga SL, et al. It is valid to dilate native aortic coartation with a balloon catheter. Int J Cardiol 1990; 27: 311-6.

4. Fontes VF, Pedra CA, Pedra SR, et al. Experiência inicial no fechamento percutâneo da comunicação interatrial com a prótese de Amplatzer. Arq Bras Cardiol 1998; 70: 147-53.

5. Friedli B, Oberhansli I, Faidutti B. Interventional catheterization in surgically treated patients with congenital heart disease. Thorac Cardiovasc Surg 2000; 48: 319-22.

6. Haddad J, Secches A, Finzi L, et al. Fechamento transvenoso percutâneo da comunicação interatrial com prótese de Sideris. Arq Bras Cardiol 1996; 67: 17-22.
7. Jacob LJB, Machado NCS, Coelho WMC, et al. Tratamento de estenose subaórtica em membrana por cateter-balão. Arq Bras Cardiol 1998; 70: 25-8.

8. Kachaner J. The best in 2000 on pediatric cardiology. Arch Mal Coeur Vaiss 2001; 94: 57-63.

9. Kreutzer J. Transcatheter intervention in the neonate with congenital heart disease. Clin Perinatol 2001; 28: 137-57.

10. Losay J, Petit J, Lambert V, et al. Percutaneous closure with Amplatzer device is a safe and efficient alternative to surgery in adults with large atrial septal defects. Am Heart J 2001; 142: 544-8.

11. Qureshi SA, Redington AN, Wren C, et al. Recommendations of the British Paediatric Cardiac Association for therapeutic cardiac catheterization in congenital cardiac disease. Cardiol Young 2000; 10: 649-67.

12. Vogel M, BergerF, Dahnert I, Ewert P, Lange PE. Treatment of atrial septal defects in symptomatic children aged less than 2 years of age using the Amplatzer septal occluder. Cardiol Young 2000; 10: 534-7.

13. Waight DJ, Hijazi ZM. Pediatric interventional cardiology: The cardiologist's role and relationship with pediatric cardiothoracic surgery. Adv Card Surg 2001; 13: 143-67. 